\section{Phosphorylation of the PRC2 component Ezh2 is cell cycle-regulated and up-regulates its binding to ncRNA}

\author{
Syuzo Kaneko, ${ }^{1,2,4}$ Gang Li, ${ }^{1,2,4}$ Jinsook Son, ${ }^{2}$ \\ Chong-Feng $\mathrm{Xu}^{3}{ }^{3}$ Raphael Margueron, ${ }^{5}$ \\ Thomas A. Neubert, ${ }^{3}$ and Danny Reinberg ${ }^{1,2,6}$ \\ ${ }^{1}$ Howard Hughes Medical Institute, New York University \\ School of Medicine, New York, New York 10016, USA; \\ ${ }^{2}$ Department of Biochemistry, New York University School of \\ Medicine, New York, New York 10016, USA; ${ }^{3}$ Department of \\ Pharmacology, Kimmel Center for Biology and Medicine, \\ Skirball Institute, New York University School of Medicine, \\ New York, New York 10016, USA
}

Ezh2 functions as a histone H3 Lys 27 (H3K27) methyltransferase when comprising the Polycomb-Repressive Complex 2 (PRC2). Trimethylation of H3K27 (H3K27me3) correlates with transcriptionally repressed chromatin. The means by which PRC2 targets specific chromatin regions is currently unclear, but noncoding RNAs (ncRNAs) have been shown to interact with PRC2 and may facilitate its recruitment to some target genes. Here we show that Ezh2 interacts with HOTAIR and Xist. Ezh2 is phosphorylated by cyclin-dependent kinase 1 (CDK1) at threonine residues 345 and 487 in a cell cycle-dependent manner. A phosphomimic at residue 345 increased HOTAIR ncRNA binding to Ezh2, while the phospho-mimic at residue 487 was ineffectual. An Ezh2 domain comprising T345 was found to be important for binding to HOTAIR and the 5 ' end of Xist.

Supplemental material is available at http://www.genesdev.org.

Received August 18, 2010; revised version accepted October 19, 2010.

The Polycomb-Repressive Complex 2 (PRC2) core components Ezh2, Eed, Suz12, and RbAp46/48 are members of the family of Polycomb Group (PcG) proteins that are key to coordinating gene repression during development and in adulthood in multicellular organisms (Kennison 1995). The means by which PRC2 targets chromatin domains in mammals is still not entirely resolved, however, and likely involves more than one pathway. Recent studies showed that the Jumonji C-containing protein Jarid2 associates with PRC2, and this association enables both Jarid2 and PRC2 to access their respective target genes in embryonic stem (ES) cells (Peng et al. 2009; Shen et al.

[Keywords: PRC2; Ezh2; phosphorylation; noncoding RNA]

${ }^{4}$ These authors contributed equally to this work.

${ }^{5}$ Present address: Research Center Unité de Génétique et Biologie du Développement, Curie Institute, 75248 Paris Cedex 05, France.

${ }^{6}$ Corresponding author.

E-MAIL reinbd01@nyumc.org; FAX (212) 263-9040.

Article is online at http://www.genesdev.org/cgi/doi/10.1101/gad.1983810. Freely available online through the Genes \& Development Open Access option.
2009; Landeira et al. 2010; Li et al. 2010; Pasini et al. 2010). In addition, several noncoding RNAs (ncRNAs) have been found to bind PRC2 (Zhao et al. 2008; Kanhere et al. 2010; Tsai et al. 2010), one of which, HOTAIR, is expressed from the HOXC cluster but recruits PRC2 to the HOXD cluster, thereby regulating transcriptional repression of these genes in trans (Rinn et al. 2007; Bonasio et al. 2010). Additionally, the ncRNA Xist, which is expressed from the female $\mathrm{X}$ chromosome directed to undergo $\mathrm{X}$ inactivation, was suggested to bind to and recruit the PRC2 complex in cis.

Although post-translational modifications (PTMs) involving phosphorylation and sumoylation have been reported for PRC2 components (for review, see Morey and Helin 2010), their functional importance is largely unknown. Phosphoproteomic analyses in the case of Ezh2 have identified phospho-serine and phospho-threonine residues at T339, S362, S363, S366, T367, S380, and T487 (see Fig. 1A; Beausoleil et al. 2004; Molina et al. 2007; Dephoure et al. 2008; Brill et al. 2009; Chen et al. 2009; Mayya et al. 2009|. One report demonstrated that Ser 21 of Ezh2 can be phosphorylated by AKT, and suggested that the phospho-mimic S21D inhibited PRC2mediated H3K27 trimethylation and gene silencing (Cha et al. 2005).

Here we show that a novel site, Thr 345 of mouse Ezh2, is phosphorylated in a cell cycle-dependent manner, and that the phospho-mimic T345D enhances Ezh2 affinity for HOTAIR ncRNA binding. We found that T345 resides within a domain important for Ezh2 interaction with HOTAIR and the $5^{\prime}$-RepA repeats of Xist.

\section{Results and Discussion}

\section{Ezh2 is phosphorylated in vivo at Thr 345}

In light of the proteomic results regarding the sites of Ezh2 phosphorylation (Fig. 1A), we used an unbiased approach to identify sites at which Ezh2 is phosphorylated in the G2/M phase of the cell cycle. Nuclear extracts (NEs) derived from 293F cells stably expressing Flag-tagged mouse Ezh2 (Flag-Ezh2) and arrested at the G2/M phase of the cell cycle were used to affinity-purify Flag-Ezh2. Flag-Ezh2 was then visualized by silver staining after SDS-PAGE and mass spectrometric analysis were performed on the purified protein. This analysis did not give evidence of the presence of phosphorylated Ser 21, reported previously (Cha et al. 2005). However, three phosphorylated threonine residues were identified (Supplemental Fig. 1). Phosphorylation at T339 and T487 was reported previously (Beausoleil et al. 2004; Dephoure et al. 2008), while T345 is a novel site. Of note, the amino acid sequences surrounding the two phosphorylated threonine residues T345 and T487 were consistent with a consensus motif recognized by cyclin-dependent kinases (CDKs) (Fig. 1A).

To test if Ezh2 could be targeted by CDKs, we first isolated GST fusion fragments of Ezh2 (Fig. 1B) containing either its $\mathrm{N}$ terminus with T345 (GST-Ezh2-N, 1-370) or its C terminus (GST-Ezh2-C, 371-746) with T487, as well as similar versions in which each of these threonine residues was substituted independently with alanine. Also, threonine residues at positions 302, 305, and 367 in the $\mathrm{N}$-terminal fragment and 416 in the $\mathrm{C}$ terminus were substituted independently with alanine. The alanine 
A

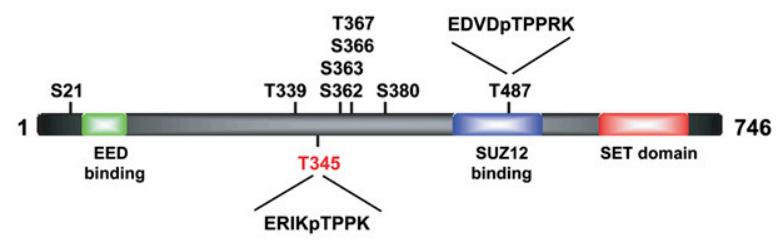

B

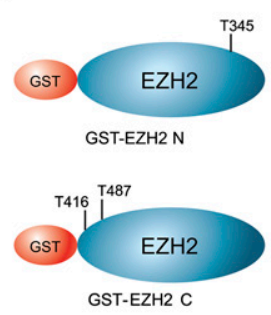

D

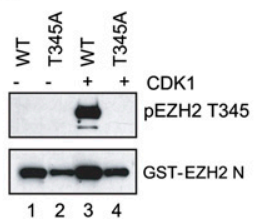

C

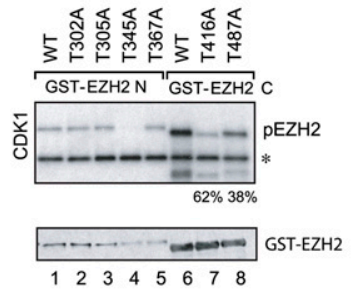

E

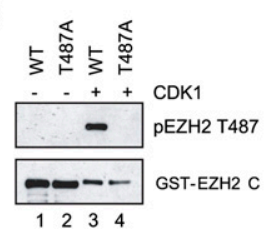

Figure 1. CDK1 phosphorylates Ezh2 in vitro. (A) Phosphorylation sites of mouse Ezh2. Previously identified phosphorylation sites are shown above the schematic (black letters). A newly identified phosphorylation site (T345 residue) is shown below the schematic (red letter). The amino acid sequences surrounding the two phosphorylated threonine residues T345 and T487 are shown. Eed- and SUZ12-binding sites and the SET domain are indicated. (B) Schematic of the GST fusion proteins containing truncated Ezh2 ([GSTEzh2 N] N-terminal-truncated; [GST-Ezh2 C] C-terminal-truncated) used for in vitro kinase assays. Phosphorylated threonine sites are indicated. $(C$, top panel) In vitro CDK1 kinase assays containing $\left[\gamma^{-32} \mathrm{P}\right]$ ATP and the single mutant Ezh2 proteins are indicated on top. (Bottom panel) Input proteins were probed with $\alpha$-GST antibody. The asterisk indicates auto-phosphorylated cyclin B. $(D, E)$ As in $B$, but using cold ATP. Phosphorylated Ezh2 was detected by immunoblot using phospho-specific antibodies.

substitution mutants of the Ezh2 termini were then tested for their ability to be phosphorylated in vitro in the presence of CDK1 and radiolabeled $\gamma$-ATP (Fig. 1C). CDK1 exhibited activity on Ezh2 as substrate with phosphorylation of the $\mathrm{N}$ terminus of Ezh2 requiring T345. CDK1 was also active on the C-terminal fragment of Ezh2 at both T416 and T487. Consistent with the absence of evidence for T302 and T305 phosphorylation in vivo, alanine substitution mutants at these sites were ineffectual in vitro. Thus the T345 and T487 residues identified as sites of phosphorylation in vivo (see above) are targeted by CDK1 in vitro. Of note, we did not observe significant phosphorylation of T345 and T487 residues by CDK2 in vitro (data not shown).

\section{Ezh2 phosphorylation at Thr 345 and Thr 487 is cell cycle-regulated}

Given that Ezh2 is a substrate of cell cycle-regulated CDKs, and that the Ezh2 preparation with which we identified phospho-T345 and phospho-T487 was derived specifically from cells arrested in G2/M, we next sought to follow the phosphorylation status of Ezh2 at each threonine as a function of the cell cycle. To this end, we

generated antibodies specific for Ezh2 phosphorylated at T345 or T487, as evidenced by the results obtained from kinase assays performed in vitro with nonradiolabeled ATP. Antibody generated against phospho-T345 recognized the Ezh2 $\mathrm{N}$ terminus that had been added to a CDK1 kinase assay, but not its alanine substitution mutant (Fig. 1D). Similarly, antibody generated against phospho-T487 recognized the Ezh2 $\mathrm{C}$ terminus treated with CDK1, but not its alanine substitution mutant (Fig. 1E).

To further validate the specificity of each antibody, we compared their reactivity to proteins isolated from wildtype and Eed-null mutant ES cells using Western blot analyses. Eed is another component of the PRC2 complex that harbors Ezh2, and, as observed previously, its absence leads to diminished cellular levels of Ezh2, although the levels of another PRC2 component, Suz12, are not severely affected (Montgomery et al. 2005). The levels of phospho-T345 and phospho-T487 versions of Ezh2, as gauged using the newly isolated anti-phospho antibodies, accurately reflected the difference in Ezh2 levels between wild-type and Eed ${ }^{-/-}$cells (Supplemental Fig. 2A). In keeping with this, Western blot analysis using the phospho-Ezh2-specific antibodies also reflected the decreased levels of Ezh2 obtained upon treating cells with shRNA specific to Ezh2, relative to control shRNA (Supplemental Fig. 2B). Moreover, Western blot analyses of NEs from ES cells before and after treatment of the membrane with calf intestinal phosphatase (CIP) confirmed that these antibodies are specific to phosphorylated Ezh2 (Supplemental Fig. 2C). Importantly, there was no detectable cross-reactivity between our phospho-Ezh2-specific antibodies against T345 and T487, as evidenced by immunoblots using phosphorylated $\mathrm{N}$-terminal- and C-terminal-truncated Ezh2 (Supplemental Fig. 2D).

Having established the specificity of the anti-phospho Ezh2 antibodies, we next used these reagents to examine the levels of endogenous Ezh2 that is phosphorylated at T345 or T487 as a function of the cell cycle. HeLa cells were sampled every $2 \mathrm{~h}$ post-release from a double-thymidine block (Fig. 2A). Western blot analysis showed that the levels of both Ezh2 and the product of its catalytic activity, trimethylation of $\mathrm{H} 3 \mathrm{~K} 37$ (H3K27me3), were relatively constant throughout the cell cycle, as were those of tubulin, while the up-regulated levels of H3S10P validated the transition from late G2 to $M$ phase. Endogenous levels of phospho-T345 and phospho-T487, while detectable in G1 phase, were clearly up-regulated during the cell cycle. The levels of phospho-T345 were sharply elevated in $G 2 / M$, after which they declined to a basal G1 level. The increase in phospho-T487 levels was broader in range, extending from late $S$ phase through $G 2 / M$ and into the subsequent G1 phase.

These results confirmed that Ezh2 phosphorylation at both sites is cell cycle-regulated and led us to test if this regulation is dependent on CDK activity (Fig. 2B). Indeed, asynchronous cells treated with the CDK inhibitor olomoucine or roscovitine resulted in undetectable levels of phospho-T345, relative to the controls in which cells were treated with either DMSO carrier or etoposide. However, both CDK inhibitors were ineffectual in the case of phospho-T487. This result is consistent with the apparent difference in the profile of phospho-T345 and phospho-T487 up-regulation during the cell cycle, and suggests that a kinase distinct from the CDK that targets 
A

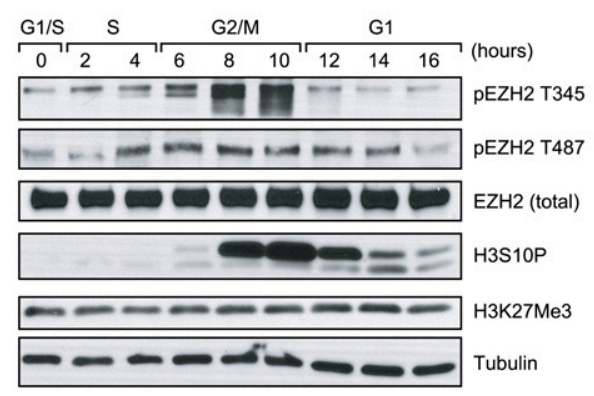

C

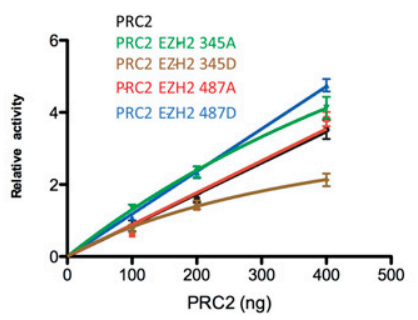

D

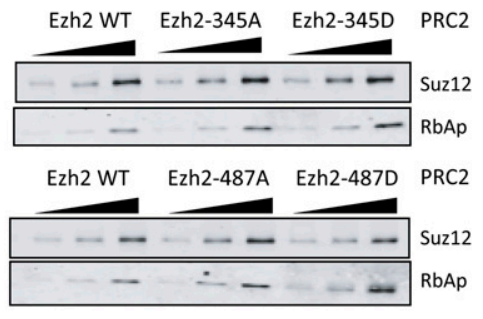

Figure 2. Ezh2 is phosphorylated by the mitotic CDK in vivo. (A) T345 and T487 of Ezh2 are phosphorylated mainly during the mitotic phase. Cells released from G1 arrest after a double-thymidine block were sampled every $2 \mathrm{~h}$, and NEs were subjected to Western analyses using the antibodies indicated on the right. $(B)$ The effect of CDK inhibitors on Ezh2 phosphorylation. HeLa cells were treated for $12 \mathrm{~h}$ with DMSO, $200 \mu \mathrm{M}$ olomoucine, $20 \mu \mathrm{M}$ roscovitine, or $50 \mu \mathrm{M}$ etoposide. NEs were subjected to Western analyses using the antibodies indicated on the right. $(C)$ In vitro HMKT assays of PRC2 complex containing the indicated Ezh2 mutants. Indicated amounts of PRC2 complexes were incubated with buffer containing $1 \mu \mathrm{g}$ of recombinant nucleosomes and $1 \mu \mathrm{M}{ }^{3} \mathrm{H}$-SAM for $60 \mathrm{~min}$. The signals were quantified by scintillation counter. $(D)$ Western blot analysis of increasing amounts of PRC2-Ezh2 mutants using the antibodies indicated on the right.

Ezh2 at Thr 345 might be responsible for its phosphorylation at Thr 487 in vivo.

The amount of Ezh2 phosphorylated at T345 and T487 is limiting in the cell

During our analyses of overall Ezh2 levels during ES cell differentiation (data not shown) and during the cell cycle, we did not observe any detectable difference in Ezh2 mobility that might reflect its being phosphorylated, suggesting that phosphorylated Ezh2 might represent but a small fraction of total Ezh2. In order to accurately gauge the amount of this fraction, we next established conditions for the quantification of Ezh2 and each of its phosphorylated versions in asynchronous cells. Supplemental Figure 3A shows that the intensities of Coomassie blue staining of equivalent amounts of recombinant GST and a GST fusion protein containing the $\mathrm{N}$-terminal fragment of Ezh2 (GST-Ezh2-N) are consistent. Calculated amounts of GST-Ezh2, as shown in the case of $500 \mathrm{fmol}$, were then compared with incremental increases in NE from ES cells in a Western blot analysis using anti-Ezh2 antibody. The results indicated that $100 \mu \mathrm{g}$ of NE contains $\sim 500 \mathrm{fmol}$ of Ezh2 protein (Supplemental Fig. 3B).

To calculate the fraction of endogenous Ezh2 that is phosphorylated in NE of ES cells, we needed to correlate the signal intensity obtained in Western blot analyses of NE when using phopsho-Ezh2-specific antibodies with that from a known amount of phospho-Ezh2. To this end, we incubated increasing amounts of GST-Ezh2-N in kinase assays containing CDK1 and radiolabeled ATP. The only product obtained was phosphorylated at Thr 345, as evidenced by the absence of radiolabel incorporation in the case of the alanine substitution mutant (Supplemental Fig. 3C). We performed a similar analysis in the case of GST-Ezh2-C that contains two potential target sites for phosphorylation (Supplemental Fig. 3D). We estimated the amount of phosphorylation at T416 and T487 by subtracting the amount of radiolabel incorporated in the case of the T487 alanine substitution mutant from the total amount of radiolabel incorporated. Given that the amounts of protein used in each case were in the linear range of radiolabeled ATP incorporation, we then calculated how much protein in a given sample was phosphorylated at a specific threonine, based on the incorporation of radiolabel derived from $\gamma$-ATP of known specific activity. We next compared the signal intensities obtained from these calculated amounts of either GST-Ezh2-N phosphorylated at T345 (Supplemental Fig. 3E) or GST-Ezh2-C phosphorylated at T416 and T487 (Supplemental Fig. 3F) with the signal intensities obtained from increasing amounts of NE from ES cells in Western blot analysis, using the appropriate phospho-Ezh2-specific antibodies. From this analysis and the amount of Ezh2 protein present in NE as determined above, we approximate that only $1 \%$ of Ezh2 is phosphorylated at T345 and only $3 \%$ is phosphorylated at T487 in asynchronous cells. Given that $\sim 30 \%$ of asynchronous cells are in the G2/M phase in HeLa cells, both T345 and T487 phosphorylation are limiting, even in G2/M phase.

The fraction of Ezh2 that is phosphorylated is relatively small, based on the calculations above. Nonetheless, given that this post-translational event is cell cycle-regulated, we reasoned that it might signify a change in the function of Ezh2 or the complex that harbors it, PRC2. To directly test the impact of Ezh2 phosphorylation on PRC2 enzymatic activity, recombinant PRC2 complex purified from Sf9 cells was assayed for histone lysine methyltransferase (HKMT) activity (Margueron et al. 2009). Neither alanine nor phospho-mimic aspartic acid substitutions at T345 and T487 of Ezh2 eliminated the HKMT activity (Fig. 2C), although PRC2 reconstituted with Ezh2 phospho-mimic aspartic acid substitutions at T345 was impaired in activity (Fig. 2C). Importantly, these Ezh2 mutants did not affect PRC2 complex formation (association of Ezh2 with Suz12, Eed, and RbAp48) (Fig. 2D). Recent results demonstrated that Jarid2 binds to Ezh2 and stimulates HKMT activity of the PRC2 complex (Li et al. 2010). None of these Ezh2 mutations (T345A, T345D, T487A, and T487D) affected Jarid2 binding to Ezh2 or Jarid2-dependent HKMT activity in vitro (data not shown).

\section{Phosphorylation of Ezh2 at Thr 345 enhances its interaction with HOTAIR ncRNA}

Previous reports indicated that Ezh2 can bind RNA species, and that PRC2 can interact with HOTAIR ncRNA 
(Zhao et al. 2008; Tsai et al. 2010). The latter interaction has been hypothesized to mediate PRC2 recruitment to some chromatin targets (Rinn et al. 2007). We next tested if PRC2 interaction with HOTAIR ncRNA is mediated through Ezh2 and, in particular, if such interaction is modulated by Ezh2 phosphorylation. Given that the characteristic negative charge of a phosphorylated residue within a protein can be mimicked by substitution with a negatively charged aspartic acid residue, we examined if Ezh2 could interact with HOTAIR ncRNA as a function of the presence of aspartic acid at residue 345 or 487 .

Figure 3 shows the results of an affinity purification of Flag-tagged full-length Ezh2 containing either an alanine (A) or an aspartic acid (D) substitution at residue 345 (Fig. $3 \mathrm{~A}$, bottom) or an alanine or aspartic acid substitution at residue 487 (Fig. 3B, bottom) from infected Sf9 cells. In each case, the resultant Ezh2 species was $>80 \%$ homogeneous. We synthesized the 5' region (1-300 nucleotdes [nt]) of HOTAIR ncRNA, which has been shown to interact with PRC2 (Tsai et al. 2010). We tested each of these Ezh2 proteins for their ability to bind radiolabeled HOTAIR ncRNA. At each protein level tested, the phospho-mimic version of residue 345 of Ezh2 resulted in elevated levels of HOTAIR ncRNA binding, relative to the case of the alanine mutant (Fig. 3A, top). On the other hand, both alanine and phospho-mimic substitutions at Ezh2 residue 487 gave rise to similar levels of binding activity for HOTAIR ncRNA (Fig. 3B, top). These results suggested that phosphorylation of Ezh2 at T345 increased Ezh2 binding to HOTAIR ncRNA.

We next attempted to identify a domain in Ezh2 that mediates its interaction with HOTAIR. Biotin-labeled, HOTAIR ncRNA (5'300 nt) was capable of binding to the N-terminal half but not the C-terminal half of Ezh2 (Fig. 3C). Furthermore, a fragment containing the N-terminal 370 amino acids of Ezh2 bound to HOTAIR; however, a fragment of Ezh2 containing the N-terminal 341 residues and therefore devoid of T345 was unable to interact with HOTAIR (Fig. 3D, top panel). Based on this finding, we deleted residues 342-368 in full-length Ezh2 and found this Ezh2 $\Delta 342-368$ mutant to be impaired in its binding to HOTAIR ncRNA, as compared with the fulllength wild-type Ezh2 (Fig. 3E). Importantly, Ezh2 $\Delta 342-$ 368 was able to interact with the other subunits of PRC2, similar to the case of wild-type Ezh2, and give rise to an unperturbed PRC2 complex. PRC2 complex was reconstituted in the case of either the Ezh2 $\Delta 342-368 \mathrm{mu}-$ tant or wild-type Ezh2 upon coinfection of baculovirus expressing the appropriate PRC2 subunits into Sf9 cells, as evidenced by Coomassie blue staining after PAGE (Fig. $3 \mathrm{~F}$, left panel) and by Western blot analysis of the isolated complex (Fig. 3F, right panel).

Zhao et al. (2008) reported that mouse Xist RepA RNA, which is located at the $5^{\prime}$ end of Xist, binds to Ezh2 and functions during initiation and spreading of X-chromosome inactivation. We analyzed whether RepA RNA can bind to Ezh2 and found this to be the case (Fig. 3D, bottom panel). Most importantly, and consistent with the results presented above, it is the $\mathrm{N}$ terminus but not the $\mathrm{C}$ terminus of Ezh2 that binds to RepA. Thus, this region of Ezh2 that we designate ncRNA-binding domain (ncRBD1) (Fig. 3G) is critical for Ezh2 binding to ncRNAs.

To probe the in vivo relevance of the findings we obtained thus far, we cotransfected a series of Flag-Ezh2 mutants and HOTAIR ncRNA into HEK293 cells (Fig. 4A).
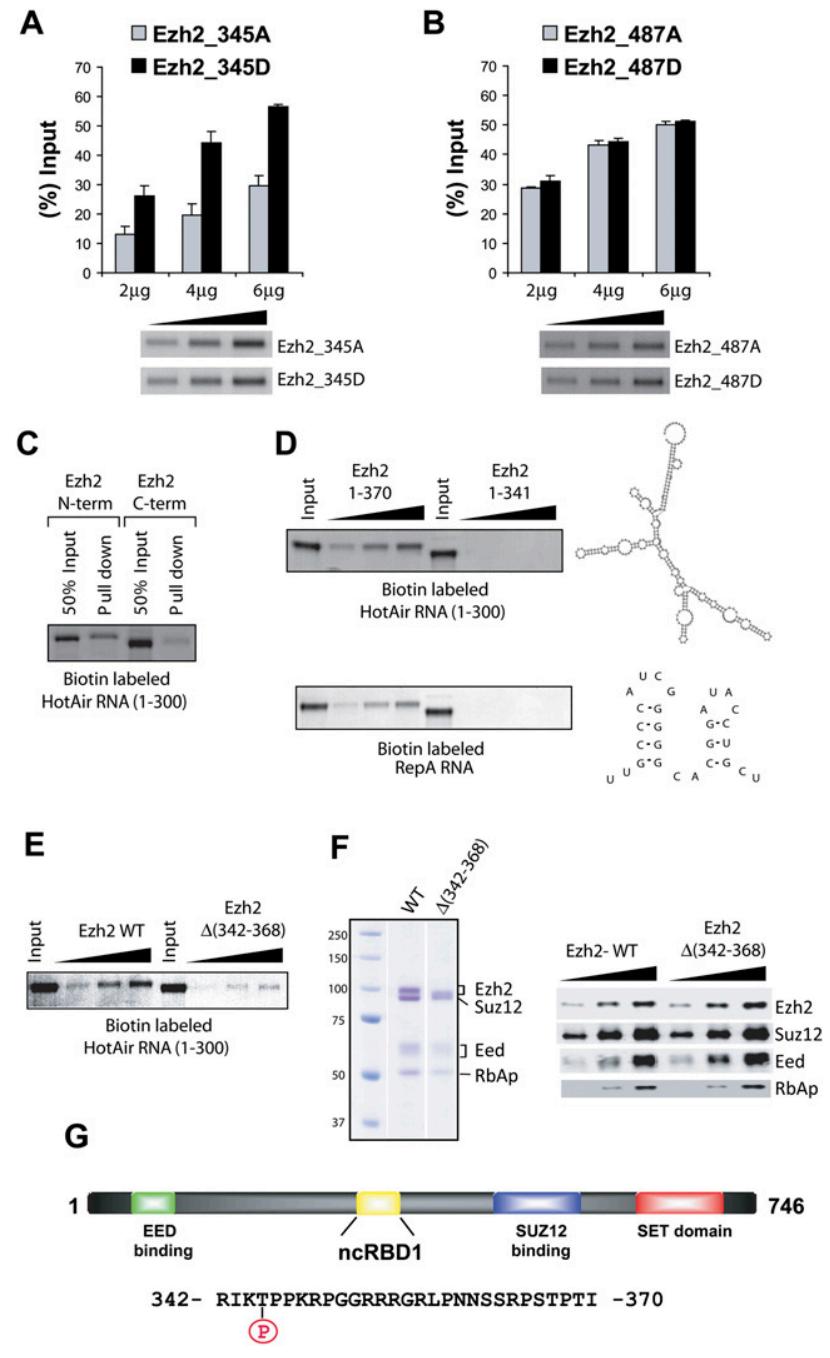

Figure 3. Phospho-mimic Ezh2 at $\mathrm{T} 345$ preferentially binds to HOTAIR ncRNA in vitro. $(A, B$, bottom panels) Coomassie blue staining of SDS-PAGE analysis of increasing amounts of Flag tag affinity-purified Ezh2 mutants, as indicated on the right, isolated from Sf9 cells. (Top panels) Bar graphs showing the quantification by scintillation counting of $\mathrm{P}^{32}-5^{\prime}$ end-labeled HOTAIR ncRNA bound to affinity-purified Ezh2. HOTAIR ncRNA was incubated with the indicated amounts of the affinity-purified Ezh2 mutants, then purified using anti-Flag M2 affinity beads. $(C)$ Biotin-labeled HOTAIR ncRNAs were incubated with $4 \mu \mathrm{g}$ of GST-Ezh2 $\mathrm{N}$ or GST-Ezh2 C proteins. Ezh2 bound to HOTAIR ncRNA was purified by Streptavidin beads and was detected by SDS-PAGE. $(D)$ As in $C$, but using increasing amounts $(2,4$, and $6 \mu \mathrm{g})$ of GST-Ezh2 1-370 or GST-Ezh2 1-341 with biotin-labeled HOTAIR ncRNA (top) or RepA RNA (bottom). The right side shows the computationally predicted structure of HOTAIR ncRNA (top) and the structure of one repeat of RepA RNA (bottom), as indicated. (E) As in $C$, but using increasing amounts $(2,4$, and $6 \mu \mathrm{g})$ of full-length Ezh2, either wild type or mutant (deletion of 342-368 amino acids), as indicated. $(F$, left panel) Coomassie blue staining of SDS-PAGE analysis of Flag tag affinity-purified PRC2 complex isolated from Sf9 cells and containing Ezh2, either wild type or deletion mutant, as indicated. (Right panel) Western analysis of increasing amounts of affinity-purified PRC2 complex containing the indicated Ezh2 component, using the antibodies indicated on the right. $(G)$ Domain structure of Ezh2. A newly identified domain (ncRBD1) required for ncRNA binding and containing the T345 phosphorylation site is shown in yellow. The amino acid sequence of ncRBD1 is shown below. 
A

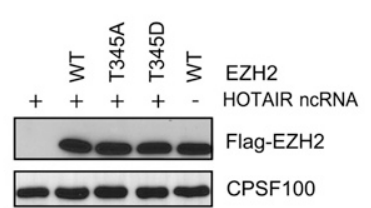

C

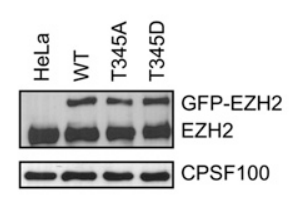

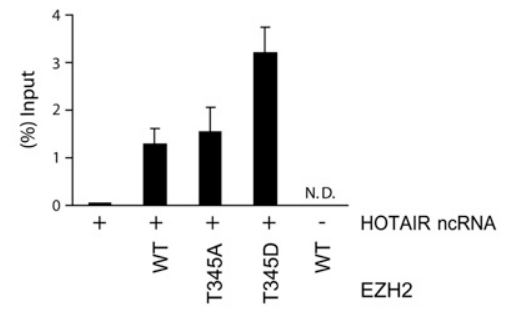

B

D

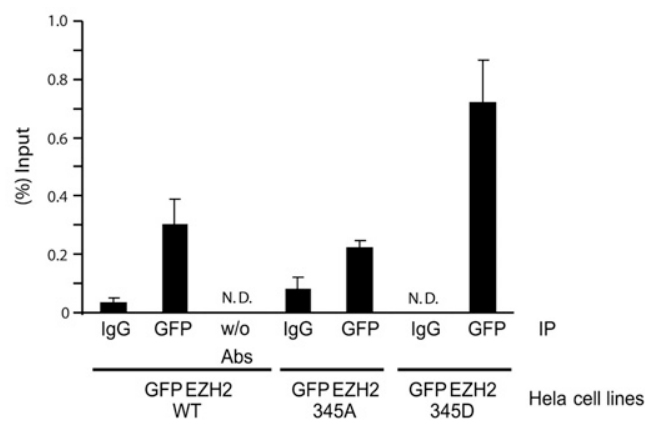

Figure 4. Phospho-mimic Ezh2 at T345 preferentially binds to HOTAIR ncRNA in vivo. (A) Cotransfection of 293 cells with the Ezh2 mutants indicated and HOTAIR ncRNA. Transiently overexpressed Ezh2 proteins were detected by anti-Flag antibody, and CPSF-100 served as a loading control. $(B)$ Bar graph of results from qRT-PCR of HOTAIR ncRNA bound to Ezh2 protein. Signals were normalized by the total amount of HOTAIR ncRNA in each case. (N.D.) Not detected. $(C)$ Western analysis of stably expressed GFP-Ezh2 mutants and endogenous Ezh2 in HeLa cells using anti-Ezh2 antibody. $(D)$ Results of RT-PCR performed on HOTAIR ncRNA bound to GFP-Ezh2 protein. PCR products were detected by qRT-PCR. (Bottom) Bar graph of qRT-PCR results for HOTAIR ncRNA with signals normalized by the total amounts of HOTAIR ncRNA in each case.

After affinity purification of Ezh2 using anti-Flag M2 antibody (Fig. 4A), RNAs bound to Flag-Ezh2 were extracted, and HOTAIR ncRNA was quantified by quantitative RT-PCR (qRT-PCR). Consistent with the binding assays performed in vitro (Fig. 3), the Ezh2 T345D mutant showed significantly increased binding to HOTAIR ncRNA, relative to the case of the wild-type and the T345A mutant version of Ezh2 (Fig. 4B). These data provide evidence that, while phosphorylation of Ezh2 at T345 is limited (Supplemental Fig. 3), it facilitates HOTAIR ncRNA binding. We next generated HeLa cell lines stably expressing GFP-Ezh2 mutants. These cell lines were treated with formaldehyde, and cell lysates were immunoprecipitated with a GFP antibody (Fig. 4C; see the Materials and Methods). RNA species that were bound to GFP-Ezh2 were extracted, and endogenous HOTAIR ncRNA was detected by RT-PCR. Once again, the Ezh2 T345D mutant exhibited a significant increase in binding to endogenous HOTAIR ncRNA, relative to the case of wild type or T345A, as evidenced by qRTPCR (Fig. 4D).

\section{Concluding remarks}

The results presented herein demonstrate that PRC2 binding to HOTAIR and RepA ncRNAs is mediated through its Ezh2 component, and that phosphorylation of Ezh2-T345 up-regulates HOTAIR-binding activity. Given that phosphorylation at this site is cell cycle-regulated, we speculate that PRC2 recruitment to chromatin, mediated through

Ezh2 interaction with HOTAIR or RepA ncRNAs and presumably other ncRNAs, must be restricted to a tightly defined interval during the cell cycle (G2/M). Most importantly, our results establish that there are at least two populations of PRC2 complexes during the G2-M stages of the cell cycle. This is consistent with a model whereby PRC2 is recruited to specific genes to initiate repression as a function of its Ezh2 component being phosphorylated at T345, after which other PRC2 complexes then spread the repressing signature (H3K27me2/3) (see below). Of note, a recent study has also documented that human Ezh2 is phosphorylated at Thr 350 (murine T345) by CDK1, and, in agreement with our findings, the report shows that this modification is ineffectual with respect to the integrity of the PRC2 complex and PRC2-mediated HKMT activity (Chen et al. 2010). Instead, this study shows that mutant Ezh2 that is not subject to T350 phosphorylation results in down-regulated PRC2 recruitment, such that appropriate gene repression is thwarted. Our report demonstrates that abrogation of this phosphorylation site within Ezh2 compromises Ezh2 interaction with ncRNAs, and this may bear directly on the mechanism by which PRC2 recruitment is impaired.

We demonstrated previously that the Eed component of PRC2 binds to trimethylated histone-repressive marks, but its binding to $\mathrm{H} 3 \mathrm{~K} 27 \mathrm{me} 3$ in particular results in an allosteric effect that markedly increases the histone methyltransferase activity of its partner, Ezh2 (Margueron et al. 2009). Thus, PRC2 binding to the product of its activity increases its production of this mark. We postulate that HOTAIR and RepA ncRNAs (and other ncRNAs) recruit PRC2 to initiate repression of target genes, and that this recruitment is enhanced by Ezh2 phosphorylation at T345. This proposed mechanism is consistent with only a small percentage of Ezh2 being phosphorylated at T345. If ncRNA species are responsible for targeting PRC2 to chromatin during G2/M, we postulate that the recruited PRC2 would set the initial H3K27me3 mark. A larger number of PRC2 complexes, independent of their Ezh2 component being phosphorylated, would then propagate this mark upon their Eed component binding to the initial H3K27me3, with resultant allosteric activation of their Ezh2 activity.

An important question remaining is whether Ezh2 is the only component of PRC2 that binds to ncRNAs. Our studies established that a 30-amino-acid domain of the PRC2-associated protein Jarid2 also binds to ncRNAs (S Kaneko and D Reinberg, unpubl.). Additionally, a recent study (Kanhere et al. 2010) suggested that the Suz12 subunit of PRC2 binds to nascent transcripts. It was postulated that this binding results in the halting of RNA polymerase II. Interestingly, it was postulated that Suz12 binding to nascent transcripts requires a unique stem-loop structure on the RNA. This structure is similar to the structure of RepA (Fig. 3D), and computer analysis of the amino acid sequence of Suz12 revealed a putative domain at its $\mathrm{N}$ terminus with a predicted RNA-binding domain (S Kaneko 
and D Reinberg, unpubl.). These observations collectively suggest that $\mathrm{PRC} 2$ recruitment to its target genes is mediated by ncRNA, different species of which likely bind to different PRC2 subunits. Whether specificity or affinity of PRC2 for its target genes is regulated by PRC2 binding through its component(s) to one family of ncRNA (specificity), or whether multiple subunits of PRC2 simultaneously bind different ncRNAs or domains within a ncRNA (affinity), remains to be established. Regardless, the studies described here and those of others (Rinn et al. 2007; Zhao et al. 2008; Kanhere et al. 2010) are collectively beginning to shed light on the role of ncRNAs in mediating the recruitment of mammalian PRC2 to its target genes.

\section{Material and methods}

Mass spectrometry, antibodies, plasmids, in vitro kinase assays, protein purification, and RNA immunoprecipitation (RIP) are described in the Supplemental Material.

\section{Synthesis of ncRNAs}

Template DNA for in vitro transcription was prepared by PCR amplification with the following primers: 5'-GGATCCTAATACGACTCACTA TAGGGAGAGACTCGCCTGTGCTCTGGAG-3' and 5'-AATAAAGAC GCCCCTCCTTC-3' (HOTAIR ncRNA 1-300 nt); 5'-GGATCCTAATAC GACTCACTATAGGGAGATGTCCTCCCCGCCATTCC- ${ }^{\prime}$ and 5'-CAT $^{\prime}$ ATTTCCATCCACCAAGC-3' (partial Xist RepA containing five tandem repeats of a 28-nt sequence that fold into two stem-loop structures, 375 nt). Full methods are described in the Supplemental Material.

\section{Ezh2-ncRNA in vitro binding assays}

Binding assays using biotin-labeled ncRNAs were carried out in the presence of indicated amounts of Ezh2 proteins with Binding buffer. Ezh2 bound to ncRNAs was purified by using Dynabeads Streptavidin (Invitrogen) washed with Binding buffer. Ezh2 was detected by SDS-PAGE followed by staining with Coomassie blue. Full methods are described in the Supplemental Material.

\section{Acknowledgments}

We are grateful to Dr. Howard Chang and Dr. Jeannie Lee for providing reagents. We thank Dr. Lynne Vales for discussion and help with manuscript preparation. This work was supported by grants from the $\mathrm{NIH}$ (GM64844) and HHMI.

\section{References}

Beausoleil SA, Jedrychowski M, Schwartz D, Elias JE, Villen J, Li J, Cohn MA, Cantley LC, Gygi SP. 2004. Large-scale characterization of HeLa cell nuclear phosphoproteins. Proc Natl Acad Sci 101: 1213012135 .

Bonasio R, Tu S, Reinberg D. 2010. Molecular signals of epigenetic states. Science 330: 612-616.

Brill LM, Xiong W, Lee KB, Ficarro SB, Crain A, Xu Y, Terskikh A, Snyder EY, Ding S. 2009. Phosphoproteomic analysis of human embryonic stem cells. Cell Stem Cell 5: 204-213.

Cha TL, Zhou BP, Xia W, Wu Y, Yang CC, Chen CT, Ping B, Otte AP, Hung MC. 2005. Akt-mediated phosphorylation of EZH2 suppresses methylation of lysine 27 in histone H3. Science 310: 306-310.

Chen RQ, Yang QK, Lu BW, Yi W, Cantin G, Chen YL, Fearns C, Yates JR III, Lee JD. 2009. CDC25B mediates rapamycin-induced oncogenic responses in cancer cells. Cancer Res 69: 2663-2668.

Chen S, Bohrer LR, Rai AN, Pan Y, Gan L, Zhou X, Bagchi A, Simon JA, Huang H. 2010. Cyclin-dependent kinases regulate epigenetic gene silencing through phosphorylation of EZH2. Nat Cell Biol . doi: $10.1038 / \mathrm{ncb} 2116$.
Dephoure N, Zhou C, Villen J, Beausoleil SA, Bakalarski CE, Elledge SJ, Gygi SP. 2008. A quantitative atlas of mitotic phosphorylation. Proc Natl Acad Sci 105: 10762-10767.

Kanhere A, Viiri K, Araujo CC, Rasaiyaah J, Bouwman RD, Whyte WA, Pereira CF, Brookes E, Walker K, Bell GW, et al. 2010. Short RNAs are transcribed from repressed polycomb target genes and interact with polycomb repressive complex-2. Mol Cell 38: 675-688.

Kennison JA. 1995. The Polycomb and trithorax group proteins of Drosophila: Trans-regulators of homeotic gene function. Annu Rev Genet 29: 289-303.

Landeira D, Sauer S, Poot R, Dvorkina M, Mazzarella L, Jorgensen HF, Pereira CF, Leleu M, Piccolo FM, Spivakov M, et al. 2010. Jarid2 is a PRC2 component in embryonic stem cells required for multilineage differentiation and recruitment of PRC1 and RNA Polymerase II to developmental regulators. Nat Cell Biol 12: 618-624.

Li G, Margueron R, Ku M, Chambon P, Bernstein BE, Reinberg D. 2010. Jarid2 and PRC2, partners in regulating gene expression. Genes DeV 24: $368-380$.

Margueron R, Justin N, Ohno K, Sharpe ML, Son J, Drury WJ III, Voigt P, Martin SR, Taylor WR, De Marco V, et al. 2009. Role of thepolycomb protein EED in the propagation of repressive histone marks. Nature 461: $762-767$.

Mayya V, Lundgren DH, Hwang SI, Rezaul K, Wu L, Eng JK, Rodionov V, Han DK. 2009. Quantitative phosphoproteomic analysis of $\mathrm{T}$ cell receptor signaling reveals system-wide modulation of protein-protein interactions. Sci Signal 2: ra46. doi: 10.1126/scisignal.2000007.

Molina H, Horn DM, Tang N, Mathivanan S, Pandey A. 2007. Global proteomic profiling of phosphopeptides using electron transfer dissociation tandem mass spectrometry. Proc Natl Acad Sci 104: 21992204.

Montgomery ND, Yee D, Chen A, Kalantry S, Chamberlain SJ, Otte AP, Magnuson T. 2005. The murine polycomb group protein Eed is required for global histone H3 lysine-27 methylation. Curr Biol 15: 942-947.

Morey L, Helin K. 2010. Polycomb group protein-mediated repression of transcription. Trends Biochem Sci 35: 323-332.

Pasini D, Cloos PA, Walfridsson J, Olsson L, Bukowski JP, Johansen JV, Bak M, Tommerup N, Rappsilber J, Helin K. 2010. JARID2 regulates binding of the Polycomb repressive complex 2 to target genes in ES cells. Nature 464: 306-310.

Peng JC, Valouev A, Swigut T, Zhang J, Zhao Y, Sidow A, Wysocka J. 2009. Jarid2/Jumonji coordinates control of PRC2 enzymatic activity and target gene occupancy in pluripotent cells. Cell 139: 1290-1302.

Rinn JL, Kertesz M, Wang JK, Squazzo SL, Xu X, Brugmann SA, Goodnough LH, Helms JA, Farnham PJ, Segal E, et al. 2007. Functional demarcation of active and silent chromatin domains in human HOX loci by noncoding RNAs. Cell 129: 1311-1323.

Shen X, Kim W, Fujiwara Y, Simon MD, Liu Y, Mysliwiec MR, Yuan GC, Lee Y, Orkin SH. 2009. Jumonji modulates polycomb activity and self-renewal versus differentiation of stem cells. Cell 139: 1303-1314.

Tsai MC, Manor O, Wan Y, Mosammaparast N, Wang JK, Lan F, Shi Y, Segal E, Chang HY. 2010. Long noncoding RNA as modular scaffold of histone modification complexes. Science 329: 689-693.

Zhao J, Sun BK, Erwin JA, Song JJ, Lee JT. 2008. Polycomb proteins targeted by a short repeat RNA to the mouse X chromosome. Science 322: $750-756$. 


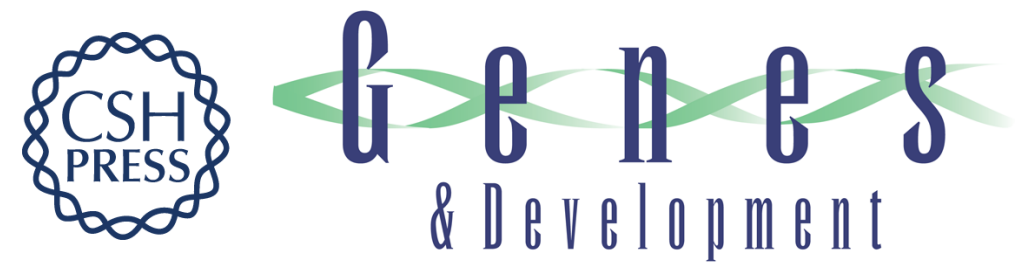

\section{Phosphorylation of the PRC2 component Ezh2 is cell cycle-regulated and up-regulates its binding to ncRNA}

Syuzo Kaneko, Gang Li, Jinsook Son, et al.

Genes Dev. 2010, 24:

Access the most recent version at doi:10.1101/gad.1983810

Supplemental
Material http://genesdev.cshlp.org/content/suppl/2010/11/29/24.23.2615.DC1

References This article cites 21 articles, 10 of which can be accessed free at: http://genesdev.cshlp.org/content/24/23/2615.full.html\#ref-list-1

License Freely available online through the Genes \& Development Open Access option.

Email Alerting Receive free email alerts when new articles cite this article - sign up in the box at the top Service right corner of the article or click here.

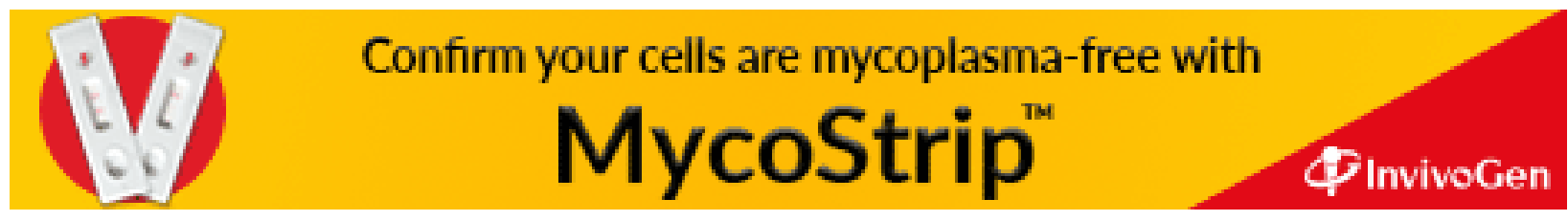

